
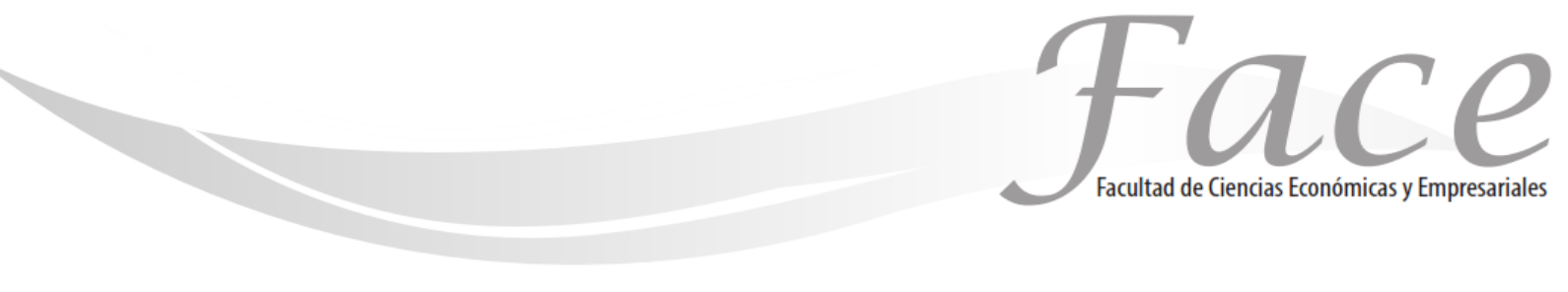

ISSN Impreso: 1794-9920 ISSN Electrónico: 2500-9338

Volumen 18-N²

Año 2012

Págs. 5- 14

\title{
ESTRATEGIAS PARA EL FOMENTO DE LA MENTALIDAD Y CULTURA EMPRENDEDORA EN LOS ESTUDIANTES DE POSGRADO DE LA CORPORACIÓN UNIVERSITARIA MINUTO DE DIOS BUCARAMANGA *
}

\author{
Eduwin Andrés Flórez Orejuela ** \\ Enlace ORCID: https://orcid.org/0000-0002-1357-0892 \\ Edward Andrés Solano Herrera *** \\ Enlace ORCID: https:// orcid.org/0000-0003-4202-672X
}

Fecha de Recepción: 10 de Octubre 2018

Fecha de Aprobación: 28 de Diciembre 2018

\section{Resumen:}

Se presentan los resultados de una investigación que comprende la revisión de las técnicas utilizadas en el centro de emprendimiento de la Corporación universitaria minuto de Dios para fomentar el emprendimiento y la innovación en sus estudiantes de posgrado, posteriormente, se mide el clima de emprendimiento e innovación para ellos siguiendo los parámetros del Global Entrepreneurship Monitor, y de ésta manera se evidencian las principales necesidades del centro, con el fin de plantear estrategias de mejora para contribuir de gran manera con el progreso del emprendimiento en la institución y así fortalecer el espíritu emprendedor en los estudiantes de posgrado.

Palabras clave: Emprendimiento, innovación, posgrado, progreso, corporación, estrategias.

\footnotetext{
* Resultado trabajo de grado para optar por el título de Ingeniero Industrial. Universidad Santo Tomás Sede Bucaramanga.

** Mágister en Administración de Empresas de la Universidad Santo Tomás, Docente de la Corporación Universitaria Minuto de Dios Bucaramanga, director del trabajo de grado. Correo: eflorezorej@uniminuto.edu.co

*** Egresado de la Facultad de Ingeniería Industrial. Universidad Santo Tomás. Correo: andres_solano_95@hotmail.com
} 


\title{
STRATEGIES FOR THE PROMOTION OF MENTALITY AND ENTREPRENEURIAL CULTURE IN POSTGRADUATE STUDENTS OF THE UNIVERSITY CORPORATION MINUTE DE DIOS BUCARAMANGA
}

\begin{abstract}
:
The results of an investigation that includes the revision of the techniques used in the entrepreneurship center of the minute university of God to promote entrepreneurship and innovation in its postgraduate students are presented, later, the climate of entrepreneurship and innovation is measured for them following the parameters of the Global Entrepreneurship Monitor, and in this way the main needs of the center are demonstrated, with the purpose of proposing improvement strategies to contribute in a great way with the progress of entrepreneurship in the institution and thus strengthen the entrepreneurial spirit in the graduate students.
\end{abstract}

Keywords: Entrepreneurship, innovation, graduate, progress, corporation, strategies.

\section{ESTRATÉGIAS PARA A PROMOÇÃO DA MENTALIDADE E DA CULTURA EMPREENDEDORA EM PÓS-GRADUAÇÃO DA UNIVERSIDADE CORPORATIVA MINUTOS DE DIOS BUCARAMANGA}

\section{Resumo:}

Os resultados de uma investigação que inclui a revisão das técnicas utilizadas no centro de empreendedorismo da minúscula universidade de Deus para promover o empreendedorismo e a inovação em seus alunos de pós-graduação são apresentados, posteriormente, o clima de empreendedorismo e inovação é medido para eles seguirem os parâmetros do Global Entrepreneurship Monitor e, assim, são demonstradas as principais necessidades do centro, com o objetivo de propor estratégias de melhoria para contribuir de forma positiva com o avanço do empreendedorismo na instituição e, assim, fortalecer o espírito empreendedor os alunos de pós-graduação.

Palavras-chave: Empreendedorismo, inovação, pós-graduação, progresso, corporação, estratégias. 


\section{INTRODUCCIÓN:}

En los países latinoamericanos, para muchos profesionales, la única opción de obtener un ingreso que los satisfaga es mediante el desarrollo de un proyecto propio. En Colombia, en el año 2016, "la economía inició con un nivel de desempleo que rondaba alrededor del $12 \%$, por lo que resulta de suma urgencia para el país buscar alternativas de generación de empleo que permitan mejorar la calidad de vida de la población" (El Espectador, 2016). Es por esto, que hoy en día el emprendimiento ha adquirido gran importancia debido a la necesidad de muchas personas de lograr su independencia y estabilidad económica.

La Corporación Universitaria Minuto de Dios Sede Bucaramanga, conocida como Uniminuto, se ha encargado de promover el emprendimiento en sus estudiantes, lo cual se ve reflejado en la creación de la Unidad de Emprendimiento Uniminuto Cedes Bucaramanga que cree en los principios y valores sociales del emprendedor como ser humano, y que tiene como rol misional fortalecer al emprendedor a través del posicionamiento de la cultura emprendedora. También, esto se refleja en algunas asignaturas que se ven a lo largo de los programas académicos y en los proyectos de grado enfocados en este tema.

Aun así, en la Universidad Uniminuto Bucaramanga no existe un sistema en donde se hallen consolidados resultados de la información anterior, por lo que no se puede analizar con verdadera certeza los efectos obtenidos en los estudiantes para poder reforzar y mejorar las falencias y al mismo tiempo resaltar lo que se está haciendo de la manera correcta. Por esta razón, se quiere analizar la importancia que tiene el emprendimiento en la comunidad de posgrado de la Universidad Uniminuto, realizando un estudio con el fin conocer las condiciones que existen en la Universidad para el fomento de una mentalidad y cultura emprendedora.

En el desarrollo del trabajo se plantea la siguiente pregunta de investigación: ¿Cuál es el estado actual en la Corporación universitaria minuto de Dios sede Bucaramanga del clima de emprendimiento e innovación en los estudiantes de posgrado y qué estrategias deben implementarse para mejorarlo?

\section{MARCO TEÓRICO:}

\subsection{Emprendimiento}

El emprendimiento es un término de origen francés que denota la realización de alguna actividad, y consta de cuatro fases, Gestación, puesta en marcha, escalamiento y consolidación e internacionalización. (Arias \& Perez, 2014)
Según la teoría del triángulo invertido propuesta por Andy Freire, todo proceso emprendedor combina tres componentes: Idea, Capital y Emprendedor. El punto de apoyo es el emprendedor, quien necesita dos componentes adicionales para llegar: la idea de negocio con viabilidad de mercado, y el capital. Cuando un emprendimiento no es exitoso, siempre se debe a la falla de una de estas tres variables, o la combinación entre ellas. De la firmeza del emprendedor depende, en gran medida, que el modelo no se derrumbe; el emprendedor "exitoso" siempre logra el capital o el gran proyecto. Por eso el problema trascendental no es el capital ni la idea, porque emprender va más allá de una mera actitud mercantil o un conjunto de conceptos. (Gomez, 2012).

Sin embargo, Schumpeter percibía al emprendedor como una persona extraordinaria que promovía nuevas combinaciones o innovaciones. "La función de los emprendedores es reformar o revolucionar el patrón de la producción al explotar una inversión, o más comúnmente, una posibilidad técnica no probada. Hacerse cargo de estas cosas nuevas es difícil y constituye una función económica distinta, primero, porque se encuentran fuera de las actividades rutinarias que todos entienden, y en segundo lugar, porque el entorno se resiste de muchas maneras desde un simple rechazo a financiar o comprar una idea nueva, hasta el ataque físico al hombre que intenta producirlo" (Shumpeter, 1935). La naturaleza de este sistema económico no permitiría un equilibrio estático ya que sería interrumpido por los esfuerzos de los emprendedores para establecer nuevas posiciones monopólicas a través de la introducción de innovaciones. Los incentivos para estas actividades serían las ganancias o rentas monopólicas que los emprendedores recibieran (Gomez, 2012).

Por otra parte, varios autores rechazaron las ideas de Schumpeter, entre ellos Ludwin Von Mises, Freidrich Hayek e Israel Kirzner. Si bien compartían la percepción de la importancia de la contribución del emprendimiento en el proceso de desarrollo capitalista, diferían en su concepción de la naturaleza de la persona y actividad en el impacto de estos en el proceso y en la visión futura del capitalismo. Para Mises, el emprendedor es aquel que desea especular en una situación de incertidumbre, respondiendo a las señales del mercado con respecto a precios, ganancias y pérdidas (Gomez, 2012).

El conocimiento generado por esta situación aumenta con el tiempo, por lo que la incertidumbre se reduce progresivamente. Para Kirzner, el emprendedor gana por estar alerta a oportunidades que existen en situaciones inciertas, de desequilibrio. Esta alerta permite al emprendedor percibir las oportunidades antes que los demás. Más aún, el emprendimiento no está encapsulado en la mera posición de un mayor conocimiento de las oportunidades del mercado. (Gomez, 2012).

Según los austríacos, el énfasis de Schumpeter en el cual el emprendedor está empujando la economía en el sentido 
contrario al equilibrio, ayuda a promover la percepción errónea de que el emprendimiento es, de alguna manera, innecesario para entender la manera en que el mercado tiende a la posición de equilibrio (Gomez, 2012).

\subsection{Global Entrepreneurship Monitor (GEM)}

El GEM, es un programa de investigación que nació con la intención de analizar la actividad emprendedora, así como los factores que influyen en ella, en relación con la creación de empresas. Tiene como función proporcionar datos acerca de la medición de la tasa de actividad emprendedora de los países que participan. "El GEM es el mayor proyecto de investigación mundial que analiza la propensión de la población de un país para participar en las actividades emprendedoras. También, es un proyecto académico que permite tener datos armonizados comparables a nivel internacional, de manera sistemática y forma anual." (Lebendiker Fainstein, Petry, Herrera Gonzalez , \& Velásquez López, 2012).

De acuerdo a lo anterior, el GEM es un proyecto que analiza los factores que influyen en la creación de empresas y el crecimiento económico de un país, teniendo en cuenta "las aportaciones de las grandes, medianas y pequeñas empresas" (Urbano, Rojas, \& Diaz, 2007). En el mismo sentido, el GEM resalta que "el emprendimiento está compuesto por actitudes, aspiraciones y actividades personales y que están directa e indirectamente involucradas en actividades emprendedoras. A su vez, esto depende de la sofisticación y grado de innovación que haya en la economía, lo que también depende de cierto contexto político, social y cultural." (Amorós \& Poblete, 2012).

En la actualidad, existen diferentes indicadores para medir la actividad emprendedora, pero el más exitoso para la medición del emprendimiento, es el GEM, el cual ha analizado a más de 100 países desde el año 1999 y que tiene como objetivo principal medir el nivel de emprendimiento en los países para probar la relación que existe con el crecimiento económico del mismo.

\subsection{Clima de Emprendimiento}

El clima de emprendimiento se define como "el conjunto de condiciones y circunstancias que rodean un lugar específico y que afectan directa o indirectamente las intenciones emprendedoras de las personas. Además, el clima de emprendimiento debe ser visto desde una óptica sistémica en la cual inciden factores de diversa índole, tanto formales como informales." (Universidad del Pacifico, 2009).

Por otra parte, se entiende como clima de emprendimiento "la relación con el entorno educacional que rodea a un estudiante y que debería repercutir en su intención de ser emprendedor" (Liñán \& Chen, 2006).

De lo anterior, podemos concluir que el clima de emprendimiento tiene una gran repercusión en que los estudiantes tengan como intención emprender y es por esto que las universidades deben realizar un esfuerzo en mejorarlo.

\subsection{Innovación}

Josep A. Schumpeter, describió la innovación como "el motor del desarrollo económico en el sistema capitalista a partir de un proceso retroalimentado de destrucción creadora" (Rausell Köster \& Abeledo Sanchis, 2012).

Por otra parte, el Manual de Oslo afirmó que "se entiende por innovación la concepción e implantación de cambios significativos en el producto, el proceso, el marketing o la organización de la empresa con el propósito de mejorar los resultados. Los cambios innovadores se realizan mediante la aplicación de nuevos conocimientos y tecnología que pueden ser desarrollados internamente, en colaboración externa 0 adquiridos mediante servicios de asesoramiento o por compra de tecnología" (Jansa, 2010). Igualmente, en el Manual de Oslo, se especifica los principales tipos de innovación, los cuales son:

- Innovación de producto: "Aporta un bien o servicio nuevo, o significativamente mejorado, en cuanto a sus características técnicas o en cuanto a su uso u otras funcionalidades, la mejora se logra con conocimiento o tecnología, con mejoras en materiales, en componentes, o con informática integrada" (Jansa, 2010).

- Innovación de proceso: "Concepto aplicado tanto a los sectores de producción como a los de distribución. Se logra mediante cambios significativos en las técnicas, los materiales y/o los programas informáticos empleados, que tengan por objeto la disminución de los costes unitarios de producción o distribución, la mejorar la calidad, o la producción o distribución de productos nuevos o sensiblemente mejorados" (Jansa, 2010).

- Innovación de mercadotecnia: "Consiste en utilizar un método de comercialización no utilizado antes en la empresa que puede consistir en cambios significativos en diseño, envasado, posicionamiento, promoción 0 tarificación, siempre con el objetivo de aumentar las ventas. La variación en el método tiene que suponer una ruptura fundamental con lo realizado anteriormente" (Jansa, 2010).

- Innovación de organización: "Es la introducción de un nuevo método organizativo en las prácticas, la organización del lugar de trabajo o las relaciones externas de la empresa" (Jansa, 2010). 


\section{METODOLOGÍA:}

Esta investigación es de enfoque mixto con elementos cuantitativos y cualitativos, se realizó mediante un estudio descriptivo (Hernandez Sampieri, 2006), que buscó especificar la situación actual del clima de emprendimiento e innovación en la población de estudio. Usó como herramienta de medición encuestas a la comunidad de estudiantes de posgrado de la Universidad Uniminuto, mayores de 22 años de edad, residentes en la región oriente del país, principalmente en el área metropolitana de Bucaramanga, fueran hombres o mujeres de cualquier estrato socio-económico.

Además, se contó con ayuda de expertos quienes proporcionaron información sobre experiencias o puntos de vistas, en relación con los factores más importantes para el emprendimiento, en este caso la técnica que se empleó fueron los grupos focales, entendiéndose este como una técnica de recopilación de información basada en entrevistas colectivas, todo aquello con el fin de plantear las encuestas que se aplicaron a la población de estudio. De tal manera se pudo analizar cómo es y cómo se manifiesta el objetivo general del presente proyecto.

Para el desarrollo de la metodología del proyecto se definieron tres fases, la revisión técnica, aplicación de instrumentos y recolección de información y la fase analítica.

\subsection{Revisión técnica}

En primer lugar, se realizó una revisión sobre las diferentes técnicas implementadas en el centro de emprendimiento de la Universidad Uniminuto para fomentar el emprendimiento e innovación en los estudiantes de posgrado. Esta revisión se enfocó en determinar la situación actual del centro de emprendimiento, con el propósito de complementarla con la metodología del Global Entrepreneurship Monitor.

\subsection{Aplicación de instrumentos y recolección de información}

Los instrumentos fueron construidos a partir del GEM, se validaron con expertos en el área de emprendimiento y educación. Posteriormente se aplicaron de forma aleatoria.

\subsection{Fase analítica}

Esta fase comprendió las siguientes actividades:

- Disposición y transformación de datos.

- Obtención de las conclusiones.

Se identificaron y clasificaron los datos obtenidos por el instrumento utilizado (Encuesta) respecto a los objetivos establecidos. Una vez realizadas estas actividades, se realizó el estudio de los resultados obtenidos, de la investigación realizada y de las conclusiones obtenidas. Los datos obtenidos se analizaron, mediante la utilización del software SPSS lo que permitió evidenciar cuál es el nivel de emprendimiento e innovación de los estudiantes de posgrado de la Corporación universitaria minuto de Dios sede Bucaramanga.

\section{RESULTADOS Y DISCUSIÓN:}

\subsection{Revisión técnica}

La Corporación Universitaria Minuto de Dios es una universidad con aproximadamente 25 años de experiencia en el mercado, actualmente tiene más de 10 sedes en los diferentes departamentos de Colombia.

En la sede de la ciudad de Bucaramanga se brinda a sus estudiantes diferentes programas académicos clasificados en postgrados, universitarios y tecnologías y estos a su vez se dividen en maestrías, especializaciones, universitarios, tecnologías, técnico laboral y técnico profesional.

Se analizaron las diferentes técnicas implementadas en su centro de emprendimiento para fomentar el emprendimiento e innovación en los estudiantes de posgrado.

Actualmente Uniminuto maneja tres especializaciones para los programas de postgrado, especialización en gerencia de proyectos, especialización en gerencia de riesgos laborales y por último, especialización en gerencia financiera, de las cuales se analizaron las técnicas que maneja el centro de emprendimiento para las dos primeras especializaciones.

En la especialización gerencia en riesgos laborales durante el plan de estudios se ven un total de veintidós créditos con una duración de dos periodos académicos y se clasifican en los siguientes componentes, general básico en el cual se ven cuatro materias, componente disciplinar específico en el cual se ve cinco materias y por último componente investigativo en el cual se ven dos materias, los componentes suman un total de once materias de las cuales ninguna tiene relación directa con los parámetros del Global Entrepreneurship Monitor (GEM) para la generación de estrategias que fomenten una mentalidad y cultura emprendedora pero a su vez en algunas materias se ven temas relacionados al emprendimiento e innovación. También en esta especialización la universidad da la opción de grado por medio de un proyecto innovador o emprendedor y comparte con sus alumnos diferentes herramientas para incrementar la cultura emprendedora.

En la especialización gerencia en proyectos durante el plan de estudios se ven un total de veinticuatro créditos con una duración de dos periodos académicos y se clasifican en los 
siguientes componente, general básico en el cual se ven cuatro materias, componente disciplinar especifico en el cual se ven seis materias y por último componente investigativo en el cual se ven dos materias, dando así un total de doce materias.

En el componente disciplinar específico las materias formulación de proyectos, evaluación del proyecto y ejecución del proyecto manejan una relación directa con los parámetros del Global Entrepreneurship Monitor (GEM) para la generación de estrategias que fomenten una mentalidad y cultura emprendedora, los demás componentes no manejan ninguna relación, pero se ven algunos temas en las diferentes materias vinculados al emprendimiento y la innovación. También en esta especialización la universidad da la opción de grado por medio de proyectos relacionados al emprendimiento y a su vez comparte herramientas para incentivar la cultura emprendedora.

\subsection{Aplicación de instrumentos y recolección de información}

Para el desarrollo de la encuesta se hizo una amplia investigación acerca del Global Entrepreneurship Monitor (GEM) y los programas de postgrado de la Corporación Universitaria Minuto de Dios seccional Bucaramanga, también se contó con la orientación y aprobación de algunos docentes de los programas de postgrado de Uniminuto, los cuales ofrecieron su experiencia en el área de emprendimiento. Todo aquello con el propósito de elaborar la encuesta relacionada al objeto de estudio.

La encuesta se aplicó a los alumnos de las especializaciones Gerencia en Riesgos Laborales y Gerencia en Proyectos, correspondientes a los programas de postgrado de la Corporación Universitaria Minuto de Dios seccional Bucaramanga.

Se elaboraron dos encuestas, la primera para el programa especialización en gerencia en riesgos laborales y la segunda para el programa especialización en gerencia de proyectos, Los tipos de preguntas que se usaron en el cuestionario fueron de selección múltiple y de escalas de valoración.

La encuesta se realizó a una muestra de 117 estudiantes pertenecientes a los programas de postgrado de la Corporación Universitaria Minuto de Dios seccional Bucaramanga.

La recolección de datos se hizo mediante algunas visitas en las sedes que maneja la Corporación Universitaria Minuto de Dios para los programas de postgrado.

\subsection{Fase analítica \\ 1.6.1. Encuesta para el programa gerencia en riesgos laborales}

La encuesta realizada estaba compuesta por un total de 14 preguntas de tipo ordinal, escala y nominal, fue contestada por 48 estudiantes que actualmente están cursando la especialización.

- Aspectos importantes: El $60 \%$ de las personas encuestadas consideran de vital importancia conocer los programas de redes y apoyo para emprendedores como una opción a tener en cuenta durante su especialización.

- Convenios: El 96\% de la población encuestada indicó que para la Universidad es fundamental concretar convenios con entidades que brinden formación y financiamiento a los que quieran emprender.

Por medio de la prueba estadística de Pearson, se analizaron los grupos "Si me parece importante" y "No me parece importante" de la pregunta sobre los "Convenios" respecto a las variables de la pregunta sobre los "Aspectos importantes". Se plantearon dos hipótesis, la nula que hace referencia a la igualdad entre grupos y se acepta cuando el nivel de significancia es superior a 0,05 y la alternativa que hace referencia a la desigualdad entre estos y se acepta cuando el nivel de significancia es inferior a 0,05. De acuerdo a la prueba Chi-cuadrado de Pearson se pudo concluir que, si hay relación entre los grupos, lo que indica que sin importar el grupo que se haya escogido los estudiantes tienden a optar por la misma variable como opción de respuesta.

- Temas: De acuerdo al análisis estadístico, se observó que la variable "Financiamiento privado y estatal para emprendedores" fue la que mayor nivel de aceptación obtuvo por los estudiantes seguida por las variables "Relación entre universidad, investigación e industria" y "Rol del investigador en el campo de emprendimiento" después como menos importantes se escogieron las variables "Emprender y fracasar" y "Experiencia de docentes emprendedores de la institución".

- Implementación electiva: El $83 \%$ de los estudiantes optaron por la implementación de una electiva en su plan de estudios con el fin de mejorar el conocimiento y la cultura emprendedora del plan de estudios.

Se analizaron los grupos "Si" y "No" de la pregunta sobre "Temas" por medio de la prueba kolmogorov-smirnov y shapiro-wilk en la cual se plantearon dos hipótesis, la nula la cual hace referencia a una distribución normal y la alternativa la cual hace referencia a una distribución no paramétrica. De acuerdo a la prueba anterior todas las variables analizadas tienen una distribución normal.

- Nivel de las materias: Para el $41,7 \%$ de las personas encuestadas, el nivel que ofrecen las diferentes materias para poder desarrollar una cultura y un conocimiento emprendedor e innovador se encuentra entre el $20 \%$ y $49 \%$. 
- Perfil emprendedor: El $75 \%$ de los encuestados se consideran con un perfil emprendedor según los parámetros del GEM.

Se analizaron los grupos "Si" y "No" de la pregunta sobre el perfil del emprendedor en relación a los grupos " $80 \%$ a $100 \%$ ", "50\% a $79 \%$ ", "20\% a $49 \%$ " y "0\% a $19 \%$ " de la pregunta sobre el nivel de emprendimiento a través de la prueba de Chi-Cuadrado debido a que son datos cualitativos. Se obtuvo que no hay diferencia entre los dos grupos, lo que indica que los estudiantes que escogieron cualquiera de los cuatro grupos se consideran emprendedores.

- Importancia del GEM: De acuerdo a los análisis estadísticos se concluye que la universidad no da a conocer la importancia del GEM en el desarrollo social y económico con un nivel de aceptación de aproximadamente el $71 \%$.

- Herramientas: Aproximadamente el $70 \%$ de los estudiantes indicaron que la universidad no da a conocer las herramientas que se manejan con el fin de incrementar el emprendimiento.

- Opción de grado: De acuerdo a los resultados obtenidos se pudo afirmar que en el programa GERENCIA EN RIESGOS LABORALES la Universidad da la opción de grado por medio de un proyecto emprendedor o innovador.

- Grado por medio de un proyecto Innovador o emprendedor: Se encontró que está dividido el nivel de aceptación de los estudiantes. Aproximadamente el $50 \%$ de los encuestados tienen la intención de graduarse por medio de proyecto emprendedor 0 innovador y la otra mitad no opta por graduarse de esta manera.

A través de la prueba estadística de Pearson se realizó el análisis de las preguntas sobre las herramientas, opción de grado y grado por medio de un proyecto innovador 0 emprendedor. Se pudo concluir que todas las preguntas se relacionan.

- Crear empresa: Se encontró que la mayoría de estudiantes están dispuestos a crear empresa ya que la Universidad les brinda las herramientas necesarias, lo que refleja que es importante que la universidad brinde la formación y el apoyo adecuado en el área de emprendimiento para poder incrementar la cultura emprendedora en el estudiantado.

- Expectativas: En cuanto a los que los estudiantes esperarían en el momento de crear empresa, se encontró con mejor nivel de aprobación el enfrentarse a nuevos retos y ser sus propios jefes, luego con un grado menor de importancia se encuentra la generación de empleo y por último con el nivel más bajo de aceptación están el tener altos ingresos económicos y asumir riesgos calculados. Lo que señala que el estudiante cree que al crear una empresa se enfrentaría a nuevos retos por la falta de formación académica, así mismo el estudiantado tiene la certeza de que al ser emprendedor y no empleado se goza de mayores beneficios.

- Dificultades: Las principales dificultades para los estudiantes al crear empresa serian: falta de capital y dificultad para obtener financiamiento. Por otra parte, el miedo al fracaso, falta de formación empresarial y falta de formación sobre el emprendimiento fueron escogidas como la dificultad menos significativa a la hora de crear empresa.

- Estudiantes emprendedores: Aproximadamente el $60 \%$ de los estudiantes del programa GERENCIA EN RIESGOS LABORALES no tienen empresa y alrededor del $40 \%$ actualmente son emprendedores.

A través de la prueba kolmogorov-smirnov y shapiro-wilk se realizó el análisis de los grupos de las preguntas sobre las expectativas y estudiantes emprendedores. Se pudo concluir que todas las preguntas se relacionan.

\subsubsection{Encuesta para el programa en gerencia de proyectos}

La encuesta realizada estaba compuesta por un total de 16 preguntas de tipo nominal, ordinal y escala, fue contestada por un total de 69 estudiantes que actualmente cursan la especialización en referencia.

- Aspectos importantes: El $58 \%$ de las personas encuestadas consideran de vital importancia conocer los programas de redes y apoyo para emprendedores como una opción a tener en cuenta durante su especialización.

- Materias y objetivos del GEM: Para el $55,1 \%$ de las personas encuestadas, la relación entre las materias y los objetivos del GEM se encuentra entre el $50 \%$ y $79 \%$, por lo que es necesario fortalecer en un $20 \%$ los temas vistos en las diferentes materias con el fin de aumentar el enfoque hacia los objetivos del GEM.

Por medio de la prueba estadística Chi-Cuadrado de Pearson se analizaron las variables de las preguntas sobre los aspectos importantes y el nivel de las materias. Se encontró que no hay diferencia significativa entre las variables de los dos grupos.

- Temas: Las variables que mayor nivel de aceptación obtuvieron por los estudiantes fueron "Financiamiento privado y estatal para emprendedores" y "Rol del investigador en el campo de emprendimiento" seguida por las variables "Relación entre universidad, investigación e industria" y "Experiencia de docentes emprendedores de la institución", después como menos importantes se escogió la variable "Emprender y fracasar". 
- Créditos y horas: Gran parte de los estudiantes encuestados de la especialización están de acuerdo con los créditos y horas acreditadas a las materias por parte de la universidad.

- Implementación electiva: El $88,4 \%$ de los estudiantes aceptan que si hay una falencia en el área de emprendimiento e innovación y para su mejora piden implementar una materia con modalidad electiva para fortalecer sus conocimientos.

Se analizaron los grupos "Si" y "No" de la pregunta sobre implementación de una electiva en relación a las variables sobre los temas, por medio de la prueba kolmogorovsmirnov y shapiro-wilk en la cual se plantearon dos hipótesis. De acuerdo a la prueba anterior se puede deducir que las siguientes variables Emprender y fracasar, financiamiento para el emprendedor y experiencia del docente manejan una distribución no paramétrica y las variables relación universidad investigación e industria y rol del investigador tienen una distribución normal.

- Nivel de las materias: Para el $52,9 \%$ de las personas encuestadas, el nivel que ofrecen las diferentes materias para poder desarrollar una cultura y un conocimiento emprendedor e innovador se encuentra entre el $50 \%$ y $79 \%$.

- Perfil emprendedor: El $86 \%$ de los encuestados se consideran con un perfil emprendedor según los parámetros del GEM.

Se analizaron los grupos "Si" y "No" de la pregunta sobre el perfil del emprendedor en relación a los grupos " $80 \%$ a $100 \%$ ", "50\% a $79 \%$ ", "20\% a $49 \%$ " y "0\% a $19 \%$ " de la pregunta sobre el nivel de emprendimiento a través de la prueba estadística de Chi-Cuadrado debido a que son datos cualitativos. Se obtuvo que no hay diferencia entre los dos grupos, lo que indica que los estudiantes que la mayoría de estudiantes se consideran con un perfil emprendedor, pero estos estudiantes afirman que la universidad no ofrece una suficiente cultura ni conocimiento emprendedor e innovador en las diferentes materias que se ven para el área de emprendimiento en el programa de postgrado analizado.

- Importancia del GEM: De acuerdo a los análisis estadísticos, con una aprobación del $62 \%$ los estudiantes de la especialización GERENCIA DE PROYECTOS dicen que la universidad da a conocer la importancia que tiene el GEM en el desarrollo social y económico.

- Herramientas: El $68 \%$ de los estudiantes de postgrado afirman que la universidad si da a conocer las diferentes herramientas para incentivar la innovación y el emprendimiento.

- Opción de grado: El 84\% de los estudiantes afirman que la Universidad da la opción de grado por medio de un proyecto emprendedor 0 innovador.
- Grado por medio de un proyecto Innovador o emprendedor: El $61 \%$ de los encuestados no tienen la intención de graduarse por medio de proyecto emprendedor o innovador.

A través de la prueba estadística de Pearson se realizó el análisis de las preguntas sobre las herramientas, opción de grado y grado por medio de un proyecto innovador 0 emprendedor. Se pudo concluir que la pregunta sobre las herramientas no tiene relación con las restantes. Es decir los alumnos que saben que la universidad da a conocer y comparte la herramientas para incentivar el emprendimiento en el programa analizado no optan por graduarse por medio de un proyecto emprendedor y a su vez algunos de estos no saben que la universidad da la opción de grado por medio de un proyecto emprendedor.

- Convenios: El 95,7\% de la población encuestada indicó que es muy importante que la universidad haga convenios con entidades que brinden formación y apoyo económico al universitario.

- Crear empresa: Aproximadamente el $65 \%$ de los estudiantes encuestados del programa están dispuestos a crear empresa debido a que la universidad brinda las herramientas necesarias para su desarrollo.

- Expectativas: En cuanto a los que los estudiantes esperarían en el momento de crear empresa, se encontró con mejor nivel de aprobación ser sus propios jefes y asumir riesgos calculados, luego con un grado menor de importancia se encuentra el tener altos ingresos económicos, generar empleo, y enfrentarse a nuevos retos. Lo que señala que es demasiado probable que al crear empresa los estudiantes sean autoritarios de tomar sus propias decisiones y asumirán riesgos ya planteados con anticipación lo que brinda más seguridad a la hora de emprender.

- Dificultades: Las principales dificultades para los estudiantes al crear empresa serian: falta de capital y dificultad para obtener financiamiento. Lo que indica que la mayoría de estudiantes no quieren emprender de acuerdo a los factores económicos. También se observó que las demás variables no fueron tan importantes de acuerdo a la valoración asignada por los estudiantes, es decir, son variables con poca importancia para relacionarlas como dificultades al momento de emprender.

- Estudiantes emprendedores: Aproximadamente el 70\% de los estudiantes del programa no tienen empresa, el $7,2 \% \%$ tuvieron empresas, pero fracasaron y aproximadamente el $21 \%$ de los encuestados actualmente tienen empresa.

A través de la prueba kolmogorov-smirnov y shapirowilk se realizó el análisis de los grupos de las preguntas sobre las expectativas y estudiantes emprendedores. Se pudo deducir que no hubo similitud entre los grupos con relación al nivel de aceptación 
otorgado a las variables estudiadas, es decir en cada variable un grupo diferente tuvo un nivel de aceptación sobresaliente.

\section{CONCLUSIONES:}

A continuación, se presentarán las conclusiones obtenidas en los resultados de las encuestas realizadas para los programas de postgrado especialización en gerencia de riesgos laborales y especialización en gerencia de proyectos, todo esto con el fin de darle solución a los objetivos planteados en el presente proyecto.

1. Programa especialización de gerencia en riesgos laborales

- Respecto a la clasificación del GEM para emprendedores se obtuvo que aproximadamente el $60 \%$ de los estudiantes actualmente están emprendiendo y el $40 \%$ restante tienen la intención de emprender 0 han fracasado durante un emprendimiento.

- Es importante agregar temas a las materias del área de emprendimiento relacionados a las variables destacadas, con el fin de mejorar el conocimiento y la cultura emprendedora en el programa académico gerencia en riesgos laborales.

- se analizó la importancia que tenía en los estudiantes la implementación de convenios con entidades públicas o privadas las cuales brindarán formación académica y apoyo económico a los emprendedores, de acuerdo a los resultados en los estudios se concluyó que los estudiantes solicitan a la universidad la inclusión de programas de redes y apoyos para los emprendedores, las caulas se pueden lograr mediante la creación de convenios con entidades públicas 0 privadas que brinden una formación académica y un apoyo económico. Lo cual se relaciona con las respuestas de la pregunta número 10 la cual indica en sus análisis estadísticos que los estudiantes están que no están dispuestos a crear empresa debido a que la universidad no da a conocer y no tiene las herramientas necesarias para emprender, por lo que es importante que la universidad haga convenios con entidades que brinden formación y apoyo económico.

\section{Programa especialización de gerencia en proyectos}

- De acuerdo a los estudios previos se observó que la mayoría de los estudiantes del programa gerencia de proyectos se consideran con perfil emprendedor, pero afirman que la universidad no ofrece una suficiente cultura ni conocimiento emprendedor e innovador en las diferentes materias que se ven para el área de emprendimiento en el programa de postgrado analizado. Asimismo, consideran que en el área de emprendimiento no se da a conocer de manera completa la importación que tiene el GEM en el desarrollo socio-económico de Colombia, debido a que solo un $62 \%$ de los encuestados le dieron su aprobación, lo que expone que hay carencia en las temáticas de las materias en relación a los objetivos del GEM.

- Se pudo observar que la mayoría de los estudiantes están de acuerdo con los créditos y horas acreditadas a las materias del área del emprendimiento pertenecientes a la especialización gerencia de proyectos.

- Se observó que los estudiantes están de acuerdo con la implementación de una materia en la modalidad de electiva con el fin de mejorar el plan académico en el área de emprendimiento e innovación, en relación a la implementación de la electiva los estudiantes solicitan temas vinculados a la variable "rol del investigador en el campo del emprendimiento", de igual manera los encuestados requieren que los temas relacionados al emprendimiento sean dictados por docentes emprendedores ya que esto da una mayor confianza al estudiante a la hora de asistir a clase porque se prefieren docentes con experiencia sobre el tema brindado.

- Respecto a la clasificación del GEM para emprendedores se obtuvo que aproximadamente el $70 \%$ de los estudiantes actualmente no tiene empresa, cerca del $8 \%$ tuvieron empresas, pero fracasaron y aproximadamente el $21 \%$ actualmente tienen empresa.

- También se analizó la importancia que tenía en los estudiantes la implementación de convenios con entidades públicas o privadas las cuales brindarán formación académica y apoyo económico a los emprendedores, por lo cual se analizaron unas series de variables de las cuales se derivó que actualmente las materias ofrecidas por el programa académico no tienen relación con los objetivos del GEM, asimismo los estudiantes solicitan a la universidad programas de redes y apoyo para emprendedores ya que aproximadamente el $65 \%$ de los estudiantes encuestados del programa están dispuestos a crear empresa debido a que la universidad brinda las herramientas necesarias para su desarrollo, por lo que se observa la carencia que hay en relación a la falta de apoyo para emprendedores, de igual forma se consideró que los estudiantes del programa especialización de gerencia en proyectos les parece muy significativo que la universidad haga convenios con entidades que brinden formación y apoyo económico al universitario, por lo que se resalta su importancia. 


\section{REFERENCIAS:}

Amorós, E., \& Poblete, C. (2012). Recuperado el 10 de Agosto de 2016, de GEM. Actividad Emprendedora en Chile y el Mundo: http://negocios.udd.cl/gemchile/files/2014/10/GE M-Chile-2012-Reporte-Actividad-Emprendedorav02_04_2013-baja-bueno.pdf

Arias, M., \& Perez, R. (2014). Emprendimiento, Como comenzar una empresa con éxito. Face. Facultad de Ciencias Económicas y Empresariales. Universidad de Pamplona, 175-183.

El Espectador. (29 de Febrero de 2016). Colombia inició 2016 con un desemepleo de 11,9\%. Recuperado el 23 de Agosto de 2016, de Colombia inició 2016 con un desempleo de 11.9\%: http://www.elespectador.com/noticias/economia/c olombia-inicio-2016-un-desempleo-de-119articulo-619416

Gomez, L. (2012). TEORIAS DEL EMPRENDIMIENTO. Recuperado el 27 de Agosto de 2016, de Teorias del emprendimiento: http://aulavirtual.tecnologicocomfenalcovirtual.ed u.co/aulavirtual/pluginfile.php/520365/mod_resou rce/content/1/TEORIAS\%20DEL\%20EMPRENDI MIENTO.pdf

Hernandez Sampieri, R. (2006). Metodología de la investigación. México: Mc Graw Hill.

Jansa, U. (2010). Recuperado el 10 de Agosto de 2016, de Resumen del Manual de Oslo sobre innovación. Concepto de Innovación: http://portal.uned.es/portal/page?_pageid=93,232 80929\&_dad=portal\&_schema=PORTAL\#concep to

Lebendiker Fainstein, M., Petry, P., Herrera Gonzalez , R., \& Velásquez López, G. (2012). Recuperado el 10 de Agosto de 2016, de La situación del emprendimiento en Costa Rica: http://www.parquetec.org/images/biblioteca/gem2 012.pdf
Liñán, F., \& Chen, Y. (2006). Recuperado el 10 de Septiembre de 2016, de Testing the entrepreneurial intention model on a two country sample:

http://upacifico.edu.ec/cladea_2009/PDF1/upac0 1_submission_451.pdf

Rausell Köster, P., \& Abeledo Sanchis, R. (2012). Recuperado el 10 de Agosto de 2016, de La cultura como facotr de innovación económica y social:

http://www.uv.es/soste/pdfs/Sostenuto_Volume1_ CAST.pdf

Shumpeter, J. (1935). Análisis del cambio económico. Ensayos sobre el ciclo económico. Recuperado el 10 de Septiembre de 2016, de Análisis del cambio económico The Review of Economics Statistics, vol. XVII, n 4, mayo 1935, pp. 2-10. : http://www.eumed.net/cursecon/textos/schumpcambio.pdf

Universidad del Pacifico. (2009). El clima de emprendimiento, un determinante clave en la intención emprendedora de los estudiantes de Escuela de Negocio. Recuperado el 10 de Agosto de 2016, de http://upacifico.edu.ec/cladea_2009/PDF1/upac0 1_submission_451.pdf

Urbano, D., Rojas, A., \& Diaz, J. (30 de Julio de 2007). Recuperado el 15 de Septiembre de 2016, de ¿Hacia dónde va la investigación en el proyecto GEM?:

http://www.academia.edu/16689012/_Hacia_d\%C 3\%B3nde_va_la_investigaci\%C3\%B3n_en_el_pr oyecto_GEM 